\title{
Age-related changes in phase-space distribution of ABPM data in normotensive and hypertensive patients
}

\author{
Giorgio Recordati* \\ Centro Interuniversitario di Fisiologia Clinica ed Ipertensione e Dipartimento, Toraco Polmonare e Cardiocircolatorio, \\ Universita' degli Studi e Fondazione Ca' Granda, IRCCS Ospedale Maggiore Policlinico, Italy
}

\section{A R T I C L E I N F O}

Article history:

Received 29 July 2010

Received in revised form 16 November 2010

Accepted 7 December 2010

\section{Keywords:}

ABPM

Heart period-blood pressure relation

Autonomic Space

Ambulatory autonomic reciprocity index

AARI

Heart period variability

AARI and R-R interval variability

AARI and aging

AARI and hypertension

AASI

\begin{abstract}
A B S T R A C T
The data collected by ambulatory blood pressure monitoring have been studied in the phase-space of R-R interval and blood pressure and their individual distribution quantified by the slope of the regression line through 24-h values. This slope has been termed "ambulatory autonomic reciprocity index" and abbreviated as AARIs and AARId, the "s" and "d" indicating the relation with systolic and diastolic blood pressure respectively. Ambulatory monitoring was performed in 200 normotensive (NT: 135 females) and 200 untreated hypertensive patients (HT: 59 females). The AARIs was: NT: $-6.04 \pm 2.7$ and HT: -4.69 $\pm 2.4 \mathrm{~ms} / \mathrm{mm} \mathrm{Hg}$, respectively $(p<0.001)$; the AARId was: $-7.04 \pm 2.9$ for NT and $-5.79 \pm 2.8$ for HT subjects $(p<0.001)$. When distributed by decades of life the steepest AARIs occurred at the 20-29 decade, while the flattest at the 60-69 decade. At the 60-69 decade and above, the AARIs was similar in both groups (ANOVA o.w. NT: $p<0.001$; HT: $p<0.01$ ). AARIs and AARId were strongly correlated with 24-h variability of R-R interval, either 24-h standard deviation or coefficient of variation $(p<0.001)$, and poorly correlated with 24-h variability of blood pressure. These data suggest that the AARI, when seen in the context of the "Autonomic Space", may be viewed as a 24-h period index of centrally driven cardiovagal function. Being based on both blood pressure and heart rate measurements, the AARI may become clinically useful to address life style changes and pharmacological treatment of hypertensive patients towards optimal results.

(C) 2010 Elsevier B.V. All rights reserved.
\end{abstract}

\section{Introduction}

It has been recently described that the ambulatory blood pressure monitoring data (ABPM) may be viewed in the phase-space of $R-R$ interval over blood pressure (Recordati and Zanchetti, 2008). This space has the coordinates of the R-R interval on the ordinate axis and of blood pressure on the abscissa to index cardio-vagal function and adrenergic sympathetic drive respectively (Berntson et al., 1991, 1993, 1994a, 1994b; Bertinieri et al., 1988; Parati et al., 2000; Coats et al., 1991; Monahan, 2007). In our representation line segments have been added to connect 24-h consecutive measurements following the time order of acquisition. The ABPM data thus appear as two clouds of points and of trajectories, one set of systolic and one of diastolic values, whose orientation may be described by computing the regression line through each set of values. For a normal circadian profile this regression line is characterized by a negative correlation coefficient and is oriented diagonally from top left (night) to bottom right (day) indicating a healthy reciprocal alternation between parasympathetic (night) and

\footnotetext{
* Centro Fisiologia Clinica ed Ipertensione, Universita' degli Studi, Ospedale Maggiore, Via F. Sforza 35, 20122 Milano, Italy. Tel.: +390255033528; fax: +390250320480. E-mail address: giorgio.recordati@unimi.it.
}

sympathetic (day) central autonomic tones (Furlan et al., 1990; Recordati, 2003; Recordati and Zanchetti, 2008). When seen in the context of the "Autonomic Space" it has been named the "reciprocity vector" (Berntson et al., 1991, 1993, 1994a, 1994b; Recordati and Zanchetti, 2008). Although its orientation may depend on many complex central and peripheral factors, which have been clearly outlined in a recent review (Monahan, 2007), overall changes in the orientation of the sets of ABPM values and of the reciprocity vector may give useful information about the individual prevailing central autonomic tone in a 24-h period (Recordati and Zanchetti, 2008). In the present paper we studied the changes in slope of the reciprocity vector occurring as a function of age and of hypertension. It is known that aging, both in normotensive and hypertensive subjects, is characterized by a spontaneous progressive increase in sympathetic activity and by a progressive decline in parasympathetic activity (Low et al., 1997; Huang et al., 2007; De Meersman and Stein, 2007; Kaye and Esler, 2008). Hypertension, on the other hand, has always been recognized as a state of low vagal and high sympathetic tone (Mancia et al., 1986; Coats et al., 1991; Wallin and Charkoudian, 2007). Hence, if the slope of the reciprocity vector is a reliable index, its changes should reflect changes related to both aging and hypertension.

To verify this hypothesis ABPM has been performed on normotensive and untreated hypertensive subjects, and differences in the slope of the regressions have been evaluated either overall or by 
decades of life between the two groups. To facilitate reading and in analogy with the very well known Ambulatory Arterial Stiffness Index, AASI, (Li et al., 2006; Dolan et al., 2006) the number indicating the slope of the regression line through 24-h data has been named the" ambulatory autonomic (space) reciprocity (vector) index" and abbreviated into ambulatory autonomic reciprocity index (AARI) with an addition of a small " $s$ " (AARIs) or "d" (AARId) to indicate the slope of the regression of the R-R interval over SBP or over DBP respectively. This term indicates the effects of the circadian reciprocal alternation between vagal and sympathetic drives on the R-R interval BP relation occurring during sleep and wakefulness in a 24 -h period (Furlan et al., 1990; Recordati, 2003, Silvani, 2008).

\section{Methods}

\subsection{Study population}

The study was performed in 200 normotensive subjects and 200 untreated hypertensive patients with essential hypertension attending for the first time our outpatient hypertension hospital clinic. Patients were included if: 1) they had never been treated; 2) their clinic BP values were in the normal and in the hypertensive ranges (mean 24 ABPM higher than 130/80 or mean day ABPM above 135/ 85) (Mancia et al., 2007); 3) had no history and clinical evidence of congestive heart failure, previous myocardial infarction or coronary bypass, cardiac valve disease, stroke, type II diabetes mellitus, sleep apnea syndrome, renal insufficiency and conditions preventing technically adequate ABPM (e.g. atrial fibrillation and other major arrhythmias). Patients with secondary hypertension were excluded. Individuals with an actual increase in night blood pressure, suggestive of Parkinson disease or autonomic insufficiency, were also excluded. Clinic BP and ABPM were performed on those patients who met the inclusion criteria and gave informed consent to participate in the study. The results of other clinical testing made on hypertensive patients, such as blood sampling for routine blood chemistry and urine analysis, 12-lead electrocardiogram, echocardiography etc. have not been included in the present study.

\subsection{Clinic BP measurement}

BP was measured during the morning (from 8.00 to 12.00 ) in the outpatient clinic by a physician or a nurse with a mercury sphygmomanometer (first and fifth phases of Koroktoff sounds taken as systolic BP (SBP) and diastolic BP (DBP) respectively, after the subject had rested for $5-10 \mathrm{~min}$ in the sitting position. The average of two-three measurements was used to characterize subject's BP.

\subsection{Ambulatory blood pressure monitoring}

Spacelabs 90207 devices were used to measure arterial blood pressure values from the non-dominant arm during $24 \mathrm{~h}$, after validation of their reading against those of a mercury sphygmomanometer. Recordings started between $8.30 \mathrm{a} . \mathrm{m}$. and $2.30 \mathrm{p} . \mathrm{m}$. The monitors were set to obtain $\mathrm{BP}$ readings at $15 \mathrm{~min}$ intervals during the day (07.00-23.00 h) and at 20 min intervals during the night (23.00$07.00 \mathrm{~h}$ ) in 55 normotensive and 95 hypertensive patients; at $20 \mathrm{~min}$ intervals during both day and night periods in 144 normotensive and 97 hypertensive patients. Nine patients (one normotensive and eight hypertensive patients) had their first monitoring done outside our institution using unusual protocols, as listed in Table 1. Meeting the inclusion criteria, their ABPM data have been included in the study and retrospectively analyzed (Table 1 ). Monitorings were carried out from Monday through Friday. The subjects were asked to attend their usual daily activities, to keep still at the time of measurement and to
Table 1

Characteristics of the study population.

\begin{tabular}{|c|c|c|c|}
\hline \multirow[b]{3}{*}{ Subjects } & \multirow{2}{*}{$\frac{\mathrm{NT}}{n(\mathrm{~F})}$} & \multicolumn{2}{|l|}{ HT } \\
\hline & & $n(\mathrm{~F})$ & \\
\hline & $200(135)$ & $200(59)$ & \\
\hline ABPM protocol 15/20 & 55 & 95 & \\
\hline ABPM protocol 20/20 & 144 & 97 & \\
\hline \multirow[t]{2}{*}{ ABPM protocol other } & 1 & 8 & \\
\hline & Mean \pm SD & Mean \pm SD & $p$ \\
\hline 24-h meas $(n)$ & $73.2 \pm 8.4$ & $76.6 \pm 10.2$ & $<0.001$ \\
\hline Age (years) & $46.9 \pm 16.8$ & $47.6 \pm 13.0$ & ns \\
\hline Clinic SBP ( $\mathrm{mm} \mathrm{Hg}$ ) & $128.3 \pm 15.8$ & $149.8 \pm 13.0$ & $<0.001$ \\
\hline Clinic DBP ( $\mathrm{mm} \mathrm{Hg}$ ) & $82.7 \pm 10.1$ & $97.1 \pm 9.2$ & $<0.001$ \\
\hline Clinic HR (beats/min) & $76.0 \pm 11.5$ & $79.1 \pm 11.8$ & \\
\hline BMI $\left(\mathrm{kg} / \mathrm{m}^{2}\right)$ & $24.1 \pm 4.0$ & $25.5 \pm 3.5$ & $<0.001$ \\
\hline Smokers $(n)$ & $35(17.5 \%)$ & $29(14.5 \%)$ & \\
\hline
\end{tabular}

$\overline{n=\text { number of subjects; }(\mathrm{F})=\text { females; } \mathrm{NT}=\text { normotensive; } \mathrm{HT}=\text { hypertensive }}$ subjects; ABPM protocol=ambulatory blood pressure monitoring every $15 \mathrm{~min}$ during the day and $20 \mathrm{~min}$ at night (15/20) and every 20 min both at day and at night $(20 / 20)$; 24 -h meas = number of valid measurements in the 24-h period; $\mathrm{BMI}=$ body mass index; $p=$ for comparison between the two groups.

note in a diary the time of switching off the light at night, of waking up in the morning, of main meals and of unusual activities.

\subsection{Analysis of data}

After acquisition, each ABPM data set was graphically represented in the time domain. Then all the data were transferred, through Excell, to an analysis and graphic program, Diadem, versions 8.1 and 9.1, National Instruments (Austin, Texas). As already reported, the R-R interval between two consecutive heart beats was calculated from $\mathrm{HR}$ ( $\mathrm{R}-\mathrm{R}$ interval in $\mathrm{ms}=60,000 / \mathrm{HR}$ ) and added to the data (Recordati and Zanchetti, 2008). Each measurement in each data set was characterized by a progressive number which identified its position with respect to the time order of acquisition and by six variables measured simultaneously: time, diastolic (DBP, mm Hg), mean (MBP, $\mathrm{mm} \mathrm{Hg}$ ) and systolic (SBP, $\mathrm{mm} \mathrm{Hg}$ ) blood pressures, heart rate (HR, beats/min), and R-R interval ( $\mathrm{ms}$ ). The values of these variables were then distributed into the set of 24-h values, the daytime and nighttime sets. Each measurement was connected to the previous (except the first one) and to the following (except the last one) by a line segment. These line segments, called the phase-space trajectories, highlight the variability of phases connecting different measurements, the transitions between day and night periods and the outer boundaries of diastolic and systolic areas, thus validating the term of "phase-space domain" (Recordati and Zanchetti, 2008).

\subsection{Statistical analysis}

2.5.1. Mean, SD, coefficient of variation, confidence interval and prediction interval

All the results are reported as mean $\pm S D$.

Mean, standard deviations (SD), and coefficient of variation (CV) were calculated for R-R intervals, HR, DBP, MBP, SBP of the entire 24$\mathrm{h}$ set (24-h) and of day and night subsets, individually in each subject, altogether in the entire cohort, and in each of the eight normotensive and six hypertensive decades (Figs. 3 and 5, Table 3). Confidence and Prediction intervals have been calculated with the aid of the statistical software enclosed to the book by Altman et al., 2000.

\subsubsection{Regression through 24- $h$ values}

According to the equation of a straight line segment, $y=a+b x$, the intercept " $a$ ", the slope " $b$ " of the regression line and the strength and the direction of the relation, the magnitude and sign of the Pearson product-moment correlation coefficient " $r$ ", between all 24-h R-R 
intervals over all 24-h systolic and diastolic blood pressure values were calculated through Diadem or the statistical program Primer on Biostatistics (Glantz, 2005), in each of the 400 patients. The slope coefficient has been termed the "Ambulatory Autonomic Reciprocity Index" and abbreviated as AARIs and AARId to indicate the R-R interval/SBP or the $\mathrm{R}-\mathrm{R}$ interval/DBP relations, respectively (Figs. 1 and 2).

\subsubsection{Ambulatory Arterial Stiffness Index}

For each subject the regression slope of 24-h values of diastolic on 24-h values of systolic blood pressure was calculated. Ambulatory Arterial Stiffness Index (AASI) was computed as 1 minus the regression slope. The stiffer the arterial tree, the closer the regression slope and AASI are to 0 and 1, respectively (Li et al., 2006; Dolan et al., 2006).

\subsubsection{Overall evaluation and comparison between normotensive and} hypertensive subjects

The overall evaluation has been made on 200 NT and 200 HT subjects (Tables 1, 2 and 3). For comparison between different variables in the same group of normo- and hypertensive subjects the values of different variables were compared by paired Student's t-test. For comparison between normotensive and hypertensive groups, the values of each variable were compared by unpaired Student's t-test. Significance was defined at three standard levels: $p<0.05^{*}, p<0.01^{* *}$, and $p<0.001^{* * *}$.

\subsubsection{Comparison amongst decades of life}

All the measured variables were grouped into decades of life of 9 years age intervals, from 10-19 to 80-89 and averaged. Variable

\section{NT CS \#183 M, 44, 73, 175 $07 / 07 / 09$}

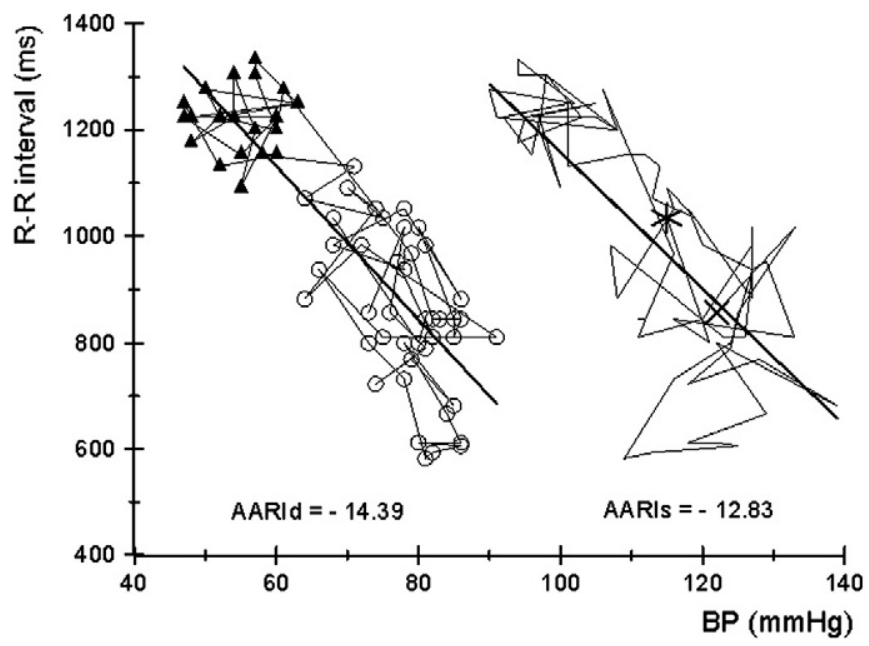

Fig. 1. Relation between R-R interval, diastolic and systolic blood pressures for the 69 measurements ( 20 min interval during both day and night) obtained in the 24-h period by ambulatory blood pressure monitoring of a normotensive 44 year old male subject (CS, \#183), $73 \mathrm{~kg}$ of weight and $175 \mathrm{~cm}$ of height, July 7, 2009. The two sets of trajectories which are generated correspond to the R-R/DBP relation on the left and to the $\mathrm{R}-\mathrm{R} / \mathrm{SBP}$ relation on the right. On the $\mathrm{R}-\mathrm{R} / \mathrm{DBP}$ relation the measurements made at night (marked by black triangles) are clearly separated from those made during the day (open circles). To make trajectories clearly visible no day and night symbols have been added to the R-R/SBP loop. Trajectories are made up by the graphic software automatically by connecting each measurement to the previous and the following one by a straight line segment. The capital X and asterisk on the R-R/SBP loop indicate the first and last measurement respectively. The straight lines crossing the two set of points from top left to bottom right are the calculated regressions for the two relations, whose slope is indicated above the abscissa by the AARId and AARIs numbers respectively.

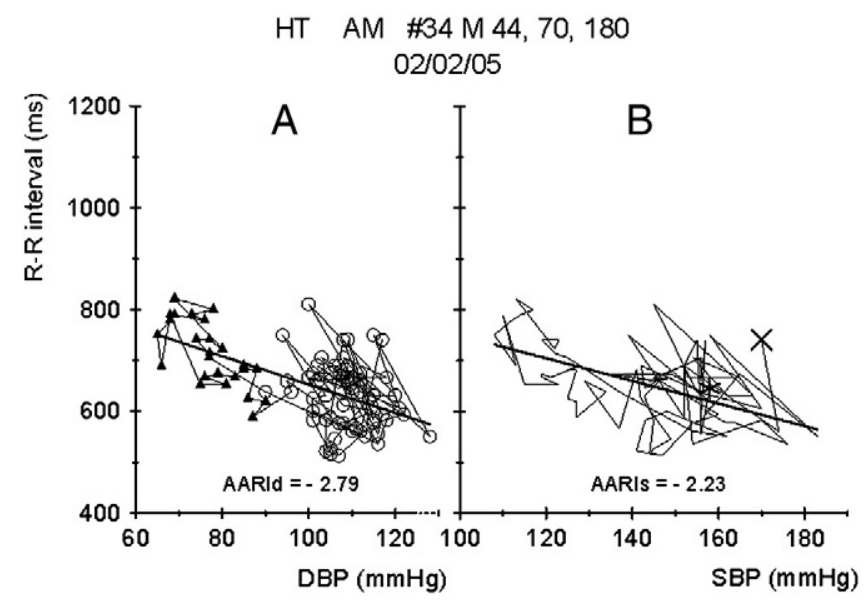

Fig. 2. Relation between R-R interval, diastolic (A) and systolic (B) blood pressures for the 88 measurements ( 15 min interval during the day and 20 at night) obtained in the 24-h period by ABPM of a hypertensive untreated 44 year old male subject (AM, \#34), $70 \mathrm{~kg}$ of weight and $180 \mathrm{~cm}$ of height, February 2, 2005. To avoid overlapping, the two loops have been drawn in different Cartesian planes. Symbols and trajectories are as in Fig. 1. AARId and AARIs indicate the slope of the regression lines which are clearly flatter than the slopes of the normotensive subject in Fig. 1.

changes between decades inside each of the groups of patients have been tested by ANOVA one way (eight decades for NT and six for HT subjects: just one normotensive subject was found in the range $0-9$, two hypertensive patients in the 10-19 decade and only one hypertensive patient in the 80-89 decade, and these decades did not enter statistical analysis). Within each group the values of the recorded variables were compared with the values of the same variable at decade 20-29 (Bonferroni correction for multiple testing, SAS Institute Inc., ver. 9.2, Cary, NC, USA). Confidence intervals for the mean have been calculated and included in Table 2. Two ways ANOVA (SAS Inst.) has been performed to verify the interaction effect between pressure and decades of life. It resulted not statistically significant and post-hoc multiple comparisons between decades of life of NT and HT subjects, therefore, has been omitted (Curran-Everett, 2000).

\subsubsection{Blood pressure and $R-R$ interval variabilities}

Variability in SBP, DBP and R-R intervals was expressed as the 24$h$ standard deviations (SD) of the average of all BP and R-R interval values measured by ABPM in the 24 -h period (Table 3 ). Coefficient of variation (CV: SD/24-h mean*100) was used to compare variability between the NT and HT groups, i.e., between different absolute levels of blood pressure and R-R interval values (Table 3and Fig. 5).

\section{Results}

\subsection{Overall evaluation}

In all 400 subjects, the nighttime set of values was positioned on the top and on the left of daytime set. In the phase-space the regressions were all directed from top to bottom and from left to right and their slopes, the term " $b x$ " of the linear equations, called the AARIs and AARId, were always negative (Recordati and Zanchetti, 2008).

Fig. 1 shows an example of a phase-space distribution of ABPM data, both R-R/DBP and R-R/SBP, for a normotensive male aged 44 and Fig. 2 for an untreated hypertensive male subject, aged 44 years. Notice that while normotensive trajectories are almost parallel to the ordinate axis, similarly to the "uncoupled parasympathetic vector", hypertensive trajectories are mainly distributed along vectors which are almost parallel to the abscissa, the "uncoupled sympathetic vector" (Fig. 2) (Berntson et al., 1991, 1993; Recordati and Zanchetti, 2008). 
Table 2

Number of subjects and variables mean \pm SD overall and by decades.

\begin{tabular}{|c|c|c|c|c|c|c|c|c|c|}
\hline \multirow{3}{*}{$n$} & \multirow{3}{*}{$\frac{\mathrm{NT}(\mathrm{F})}{\mathrm{HT}(\mathrm{F})}$} & \multirow{3}{*}{$\frac{\frac{\text { Overall }}{200(135)}}{200(59)}$} & \multirow{3}{*}{$\frac{\frac{\text { Dec } 20-29}{27(14)}}{14(4)}$} & \multirow{3}{*}{$\frac{\frac{30-39}{27(16)}}{38(12)}$} & \multirow{3}{*}{$\frac{40-49}{\frac{41(28)}{60(14)}}$} & \multirow{3}{*}{$\frac{50-59}{\frac{42(31)}{51(17)}}$} & \multirow{3}{*}{$\frac{60-69}{35(28)} \frac{25(8)}{25}$} & \multirow{3}{*}{$\frac{70-79}{11(8)} \frac{9(3)}{}$} & \multirow[t]{3}{*}{ ANOVA o.w. } \\
\hline & & & & & & & & & \\
\hline & & & & & & & & & \\
\hline \multirow[t]{4}{*}{ AARIs } & NT & $-6.04 \pm 2.7^{9 g g}$ & $-8.5 \pm 2.2$ & $-6.8 \pm 2.2$ & $-6.9 \pm 2.7$ & $-5.2 \pm 2.4^{\S}$ & $-4.0 \pm 1.9^{\S}$ & $-4.9 \pm 2.9^{\S}$ & $* * *$ \\
\hline & $95 \% \mathrm{CI}$ & $-6.4 /-5.7$ & $-9.3 /-7.6$ & $-7.7 /-6.0$ & $-7.7 /-6.0$ & $-5.9 /-4.5$ & $-4.7 /-3.4$ & $-6.8 /-2.9$ & \\
\hline & HT & $-4.69 \pm 2.4$ & $-6.2 \pm 2.4$ & $-5.4 \pm 2.0$ & $-4.8 \pm 2.6$ & $-4.0 \pm 2.6^{\S}$ & $-3.8 \pm 1.4^{\S}$ & $-3.9 \pm 1.3$ & ** \\
\hline & $95 \% \mathrm{CI}$ & $-5.0 /-4.4$ & $-7.6 /-4.8$ & $-6.1 /-4.8$ & $-5.5 /-4.2$ & $-4.8 /-3.4$ & $-4.4 /-3.3$ & $-4.9 /-3.0$ & \\
\hline \multirow[t]{4}{*}{ AARId } & NT & $-7.04 \pm 2.9^{999}$ & $-8.8 \pm 2.5$ & $-7.2 \pm 3.0$ & $-7.6 \pm 2.7$ & $-6.7 \pm 3.1^{\S}$ & $-5.3 \pm 2.3^{\S}$ & $-7.4 \pm 3.5$ & $* * *$ \\
\hline & $95 \% \mathrm{CI}$ & $-7.4 /-6.6$ & $-9.8 /-7.9$ & $-8.4 /-6.0$ & $-8.5 /-6.8$ & $-7.6 /-5.7$ & $-6.0 /-4.7$ & $-9.7 /-5.0$ & \\
\hline & HT & $-5.79 \pm 2.8$ & $-7.1 \pm 3.2$ & $-6.1 \pm 2.7$ & $-5.7 \pm 2.8$ & $-5.5 \pm 2.8$ & $-5.4 \pm 2.6$ & $-5.8 \pm 1.8$ & ns \\
\hline & $95 \% \mathrm{CI}$ & $-6.2 /-5.4$ & $-9.0 /-5.3$ & $-7.0 /-5.2$ & $-6.4 /-4.9$ & $-6.2 /-4.7$ & $-6.5 /-4.3$ & $-7.1 /-4.4$ & \\
\hline \multirow[t]{4}{*}{ AASI } & NT & $0.31 \pm 0.14$ & $0.18 \pm 0.11$ & $0.28 \pm 0.11^{\S}$ & $0.28 \pm 0.10^{\S}$ & $0.33 \pm 0.14^{\S}$ & $0.40 \pm 0.11^{\S}$ & $0.43 \pm 0.15^{\S}$ & $* * *$ \\
\hline & $95 \% \mathrm{CI}$ & $0.29 / 0.33$ & $0.14 / 0.22$ & $0.24 / 0.33$ & $0.25 / 0.31$ & $0.29 / 0.38$ & $0.36 / 0.44$ & $0.33 / 0.52$ & \\
\hline & HT & $0.30 \pm 0.13$ & $0.25 \pm 0.12$ & $0.25 \pm 0.13$ & $0.27 \pm 0.13$ & $0.35 \pm 0.12$ & $0.37 \pm 0.12$ & $0.41 \pm 0.09^{\S}$ & $* * *$ \\
\hline & $95 \% \mathrm{CI}$ & $0.28 / 0.32$ & $0.18 / 0.31$ & $0.21 / 0.29$ & $0.23 / 0.30$ & $0.31 / 0.38$ & $0.32 / 0.42$ & $0.34 / 0.48$ & \\
\hline
\end{tabular}

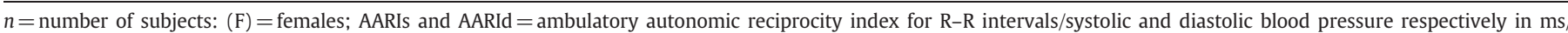

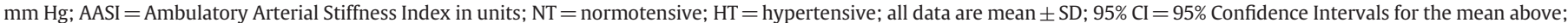

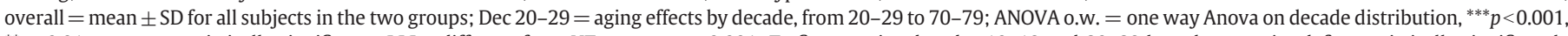

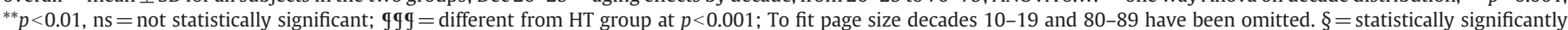

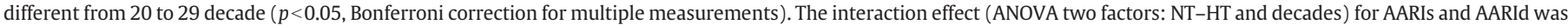
not statistically significant and post-hoc analysis for decades between NT and HT has been omitted.

In 200 normotensive (NT mean age: $46.9 \pm 16.8$ years, 135 females, $67.5 \%$ ) and 200 hypertensive patients (HT mean age: $47.6 \pm 13.0$ years, 59 females, $29.5 \%$, unpaired Student's $t$-test for age: ns, Table 1) the mean AARIs was NT: $-6.04 \pm 2.7$ and HT: $-4.69 \pm 2.4 \mathrm{~ms} / \mathrm{mm} \mathrm{Hg}$, $(p<0.001)$ (Table 2 and Fig. 4$)$ and the AARId was NT: $-7.4 \pm 2.9$ and HT: $-5.79 \pm 2.8 \mathrm{~ms} / \mathrm{mm} \mathrm{Hg}$ respectively $(p<0.001)$ (Table 2 and Fig. 4 ); the mean 24-h SBP (NT: 118.3 \pm 7.9; HT: $137.2 \pm 8.2 \mathrm{~mm} \mathrm{Hg}, p<0.001$ ) and DBP (NT: $72.6 \pm 6.7$; HT: $87.3 \pm 6.8 \mathrm{~mm} \mathrm{Hg}, p<0.001)$ and mean $24-$ h HR (NT: $72.8 \pm 8.5$; HT: $76.0 \pm 9.0, p<0.001$ ) were statistically significantly lower in normotensive than in hypertensive subjects, while the R-R interval (NT: $856.5 \pm 105.4$; HT: $817.9 \pm 100.7 \mathrm{~ms}$, $p<0.001$ ) was significantly higher in normotensive than in hypertensive patients.

In each subject the AARId was compared with the AARIs by paired Student's t-test. Overall, in both normotensive $(n=200)$ and hypertensive ( $n=200)$ groups, the mean AARId was steeper than the corresponding mean AARIs (Figs. 1 and 2, for single subjects) and this difference was highly statistically significant $(p<0.001)$.

Table 3

Variability of systolic and diastolic blood pressure and R-R interval in the 24-h period and its relation with AARIs and AARId.

\begin{tabular}{lclll}
\hline Variable & $\begin{array}{l}\text { NT } \\
\text { mean } \pm \text { SD }\end{array}$ & & $\begin{array}{l}\text { HT } \\
\text { mean } \pm \text { SD }\end{array}$ & $p$ \\
\hline 24-h SBP SD & $12.1 \pm 2.8$ & & $15.5 \pm 3.9$ & $<0.001$ \\
24-h SBP CV & $10.2 \pm 2.3$ & & $11.3 \pm 2.9$ & $<0.001$ \\
24-h DBP SD & $10.5 \pm 2.2$ & & $12.8 \pm 2.8$ & $<0.001$ \\
24-h DBP CV & $14.6 \pm 3.4$ & & $14.8 \pm 3.5$ & $\mathrm{~ns}$ \\
24-h RR SD & $129.8 \pm 35.0$ & & $120.4 \pm 37.1$ & $<0.01$ \\
24-h RR CV & $15.2 \pm 3.7$ & & $14.7 \pm 3.9$ & $\mathrm{~ns}$ \\
& & & & \\
Regression & $r$ & $p$ & $r$ & $p$ \\
\hline AARIs vs SBP24 SD & 0.1582 & $<0.05$ & 0.1549 & $<0.05$ \\
AARIs vs SBP24 CV & 0.1505 & $<0.05$ & 0.1186 & $\mathrm{~ns}$ \\
AARIs vs RR24 SD & 0.6708 & $<0.001$ & 0.6729 & $<0.001$ \\
AARIs vs RR24 CV & 0.6633 & $<0.001$ & 0.6532 & $<0.001$ \\
AARId vs DBP24 SD & 0.0532 & $\mathrm{~ns}$ & 0.1379 & $<0.05$ \\
AARId vs DBP24 CV & 0.0693 & $\mathrm{~ns}$ & 0.1483 & $\mathrm{~ns}$ \\
AARId vs RR24 SD & 0.7291 & $<0.001$ & 0.7896 & $<0.001$ \\
AARId vs RR24 CV & 0.6935 & $<0.001$ & 0.747 & $<0.001$ \\
\hline
\end{tabular}

Top panel: $\mathrm{NT}=$ normotensive; $\mathrm{HT}=$ hypertensive; $\mathrm{SD}=$ standard deviation $\mathrm{CV}=$ coefficient of variation; $p=$ for comparison between the two groups. Bottom panel: linear regressions for both NT and HT groups; $r=$ correlation coefficient; $p=$ Statistical significance of $r ; n=200$ for all the comparisons.
Overall the mean AASI for NT was $0.3068 \pm 0.1401$ and for HT $0.3043 \pm 0.135$ units $(p=n s)$.

\subsection{Decades averages}

Normotensive and hypertensive subjects have been grouped along decades of age and the number of subjects in each decade has been reported in Fig. 3D and Table 2. The results of one way analysis of variance for hemodynamic variables were as follows: NT SBP: ns, DBP: $p<0.01$, HR: ns, R-R interval: ns; HT SBP: $p<0.05$, DBP: $p<0.001$, HR: $p<0.05$, R-R interval: $p<0.05$. Two ways ANOVA (factors: NT-HT and decades) was statistically significant $(p<0.05)$ for DBP changes only, owing to the pressure difference and to the blood pressure drop which occurred in the last decade in NT (80-89) and HT (70-79) groups (Fig. 3C).

Fig. 4 shows changes occurring in AARIs (Fig. 4A) and AARId (Fig. 4B), in both normotensive and hypertensive patients distributed by decades. The steepest mean slope was reached in the 20-29 decade while the flattest in the 60-69 decade in both groups and for both AARIs and AARId.

Overall the AARIs and the AARId of NT ( $n=200)$ were steeper than those of HT ( $n=200)$ at $p<0.001$ (Fig. 4 A and B and Table 2). One way ANOVA was statistically significant for AARIs and AARId of NT $(p<0.001$, eight decades), for AARIs of HT $(p<0.01$, six decades), and not statistically significant for AARId of HT group (six decades, Table 2). In the NT and HT subjects the AARIs of 20-29 years old subjects resulted statistically significantly steeper than AARIs of the subjects in the last three decades, and in the 50-59 and 60-69 decades, respectively (Table 2). The effect of aging seems thus to be very evident on AARIs of both NT and HT subjects and on AARId of NT, while being inconsistent on AARId of HT group.

Two-way ANOVA (factors: NT-HT and decades) on AARIs for the six shared decades, 20-29 to 70-79, gave the following main effects: NT-HT $p<0.001$, decades $p<0.001$. The interaction effect of pressure and age was, however, not statistically significant $(p=0.19)$, indicating that the age declining of AARIs was similar in NT and HT groups. Two ways analysis of variance of AARIs became almost statistically significant $(p=0.062)$ when the six decades were combined into three groups: young (decades 20-29 and 30-39) middle (decades 40-49 and 50-59) and old (60-69 and 70-79), thus indicating that the interaction effect is probably underscored either by the relatively small number of subjects in each decade and by the relatively high variance of results in each decade. Because of this 


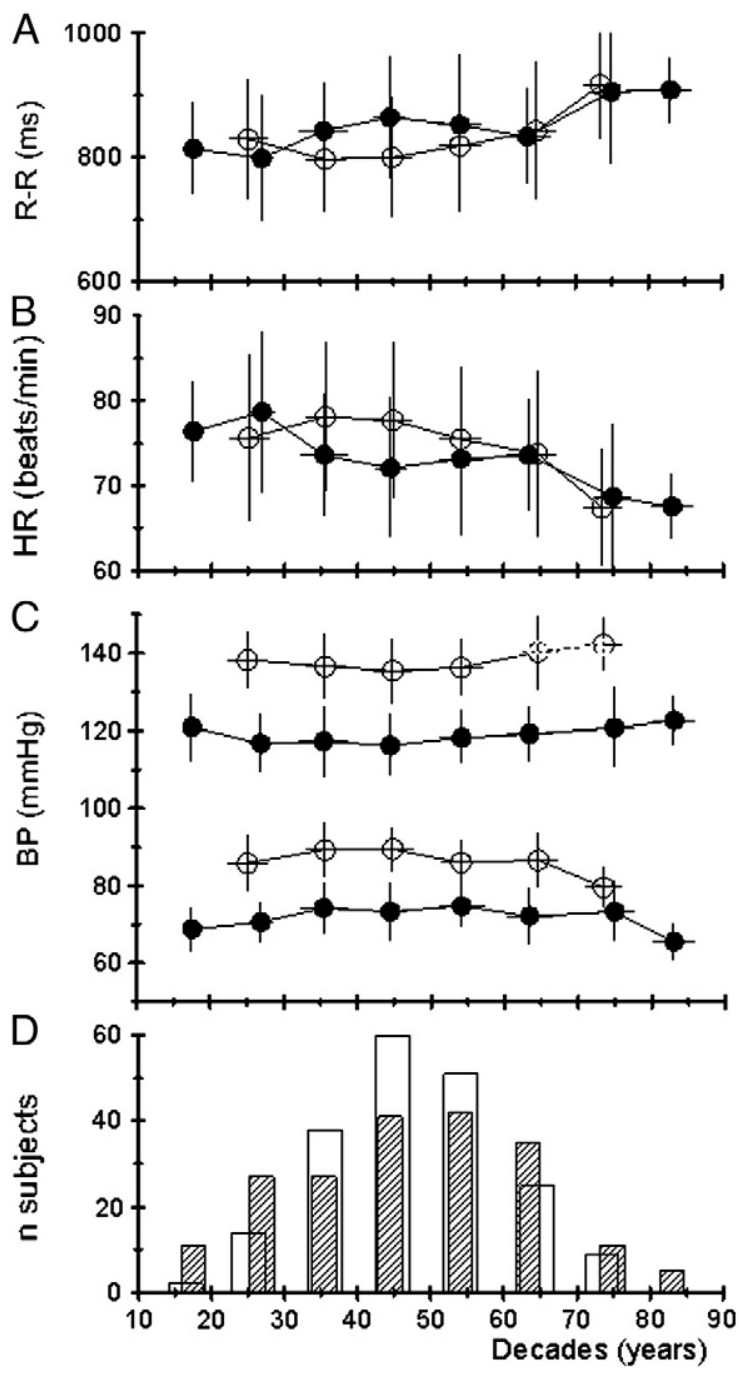

Fig. 3. Relation between age and R-R interval (A), heart rate (B), systolic and diastolic blood pressures $(C)$ and number of subjects $(D)$ in eight normotensive (from 10--19 to 80-89) and six hypertensive (from 20-29 to 70-79) decades of life. Normotensive subjects: closed circles; hypertensive subjects: open circles. Please notice the bell distribution of subjects (D) with the maximum in the 40-49 and 50-59 decades for both groups (hatched bars: normotensive subjects; open bars: hypertensive subjects). Heart rate (B) slowly declined with age and above 60 no differences between normoand hypertensive subjects have been measured. Systolic blood pressure of hypertensive subjects was always statistically significantly higher than SBP of normotensive. Diastolic blood pressure was always statistically higher than in normotensive except for the last decade, 70-79.

negative result post-hoc analysis of differences between decade means of AARIs of NT and HT subjects has been omitted (CurranEverett, 2000).

\subsection{Ambulatory Arterial Stiffness Index (AASI)}

Fig. 4C shows the decade distribution of AASI values. Overall no statistically significant differences have been found in AASI between NT vs HT subjects (Table 2). AASI increased with age from the minimum reached at the 20-29 decade in both NT and HT patients, to the maximum reached at 70-79 decade in both NT and HT subjects (ANOVA one way: $p<0.001$, for both NT and HT groups). In NT, the mean AASI value of 20-29 decade was highly statistically significantly different from the mean value of all subsequent decades $(p<0.05)$, while in HT the mean AASI value of 20-29 decade was statistically

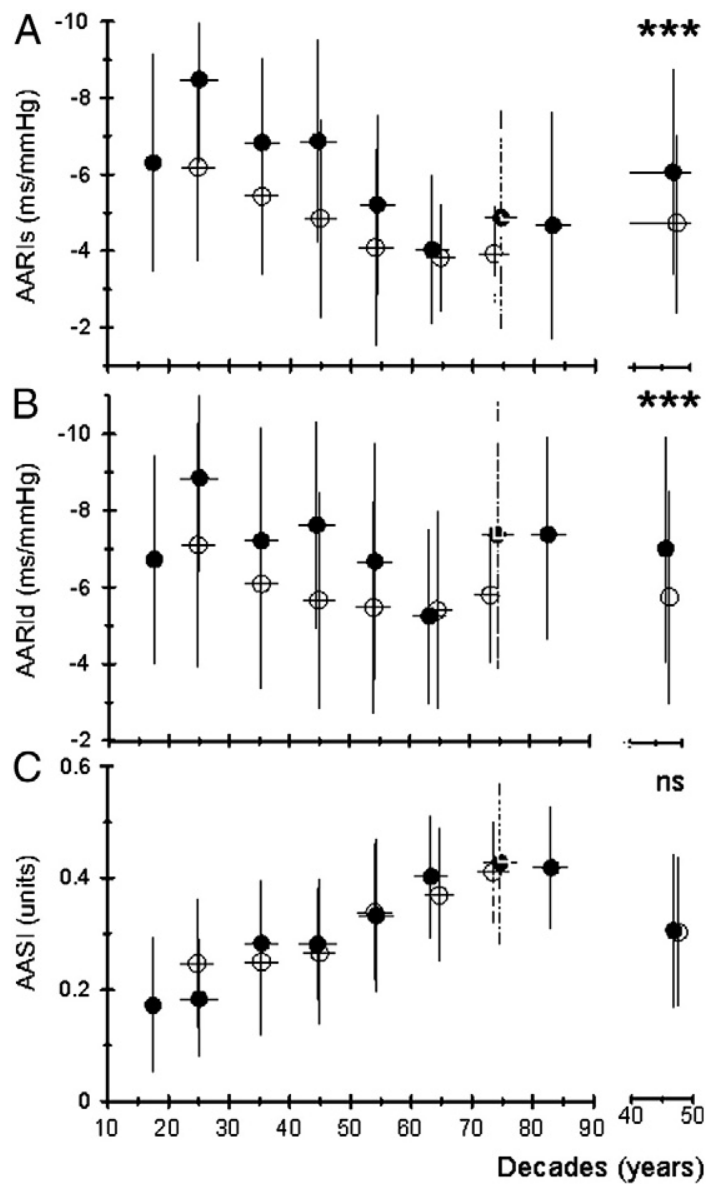

Fig. 4. Relation between age and AARIs (A), AARId (B) and AASI (C) overall and in eight normotensive and six hypertensive decades. Normotensive subjects: closed circles; hypertensive subjects: open circles. The last column on the right, with abscissa 40-50, shows the variables mean value $\pm S D$ (the overall partial SD for age has been included in panel A only) for all normotensive and hypertensive subjects. Overall the AARIs and the AARId were statistically significantly steeper in normotensive than in hypertensive subjects at the $p<0.001$ while AASI difference (C) was not statistically significant. In both normotensive and hypertensive subjects the AASI increased from the 20-29 to the 60-69 decade.

significantly lower of the mean AASI of the last decade only (70-79: $p<0.05$ ).

AASI correlated significantly with AARIs $(p<0.001)$ but not with AARId ( $p=n s)$, for both NT $(r=0.374)$ and HT $(r=0.244)$ groups. The slopes and the intercepts of the regressions of AASI over AARIs of NT and HT groups did not result statistically significantly different.

\subsection{AARI and 24-h variability of blood pressure and $R-R$ interval}

As reported in Table 3, the average SD of 24-h SBP and DBP values were statistically significantly higher and the SD of 24-h R-R interval statistically significantly lower, in the HT than in the NT group while no differences have been found in the average CV of DBP and R-R interval.

To verify whether AARI was dependent on blood pressure and/or heart rate variability the correlations between AARIs and AARId and systolic, diastolic and R-R interval variability have been calculated (Table 3 and Fig. 5). The AARIs and AARId were highly statistically correlated with the 24-h variability of R-R interval, measured as SD and CV, in both NT and HT subjects (Table 3 and Fig. 5, top panels). On the other hand the AARIs correlated in both NT and HT subjects with the 24-h variability of SBP if measured as SD, while it correlated with the 24-h variability of SBP, measured as CV, in NT subjects only (Table 3 and Fig. 5, bottom panels). No statistically significant 


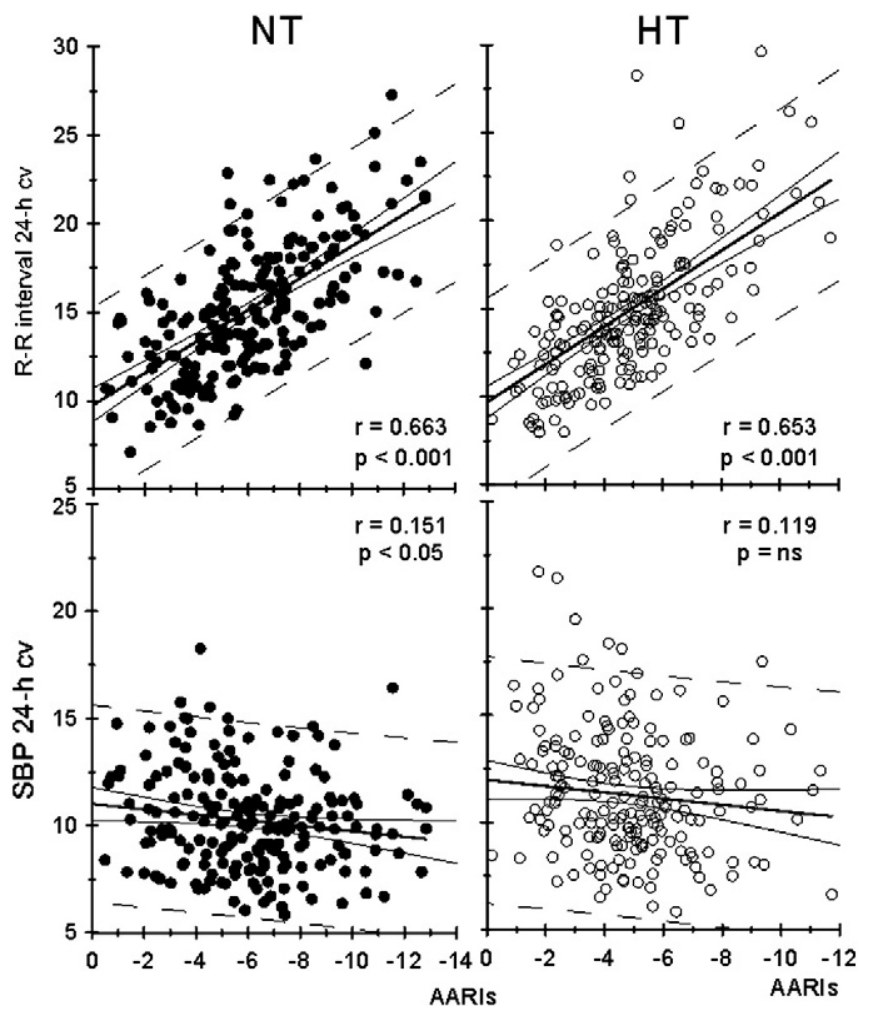

Fig. 5. Relations between AARIs and variability of R-R interval (24-h coefficient of variation, CV, top panels) and variability of SBP (24-h coefficient of variation, CV, bottom panels) for all normotensive (NT, left panels, closed circles) and hypertensive (HT, right panels, open circles) subjects. Regression coefficient and statistical significance have been calculated for $n=200$ subjects. In each panel the middle straight line is the regression between the two variables, the nearest curved lines are the $\mathrm{CI}$ for the regression, while the outermost dashed lines delimit the Prediction Interval for the regression. Please notice the strong correlation of AARIs with variability of R-R interval (that of AARId is reported in Table 3).

correlation was found between AARId and 24-h variability of DBP measured as CV (Table 3). These data indicate that AARI is strongly dependent on heart period variability and much less dependent on variability of blood pressure.

\section{Discussion}

The major findings of the present study are the following: a) the steepness of the regression slope through 24-h values of RR/SBP and of RR/DBP, termed the AARIs and AARId, reaches its maximum during the 20-29 decade in both NT and HT subjects, then declines progressively until the 60-69 decades and while the AARIs seems to remain stable thereafter, the AARId further increases in the last two decades (Fig. 4A and B); b) in both NT and HT groups the AARIs at 2029 decade is statistically significantly steeper than at 50-59 and 60-69 decades thus documenting a consistent decline with aging; c) overall, the AARIs and the AARId of the normotensive group were highly statistically significantly steeper than those of the hypertensive group; d) from 20-29 to 50-59 decades the average regression's slope were less steep in the hypertensive than in normotensive group, this difference not reaching statistical significance, however; c) both AARIs and AARId have been found to be highly statistically correlated with 24-h R-R interval variability but AARIs only has been found correlated with blood pressure variability; $d$ ) the AARIs seems a better index than AARId to describe age and hypertension effects and our discussion will be mainly concerned with AARIs changes. Although our approach is directed to study the outcome of the complex autonomic influences acting either centrally or peripherally on the cardiovascular system in a 24 -h period the interpretation of our data may take advantage of the previous studies performed by continuous intrarterial recordings of blood pressure and R-R interval in humans (Mancia et al., 1986 and Coats et al., 1991). These studies have shown that changes in slope's regression reflected reciprocal changes in $\mathrm{R}-\mathrm{R}$ interval and BP variability: while a steep slope indicated a predominant R-R interval variability, a flat slope was dependent on prevailing $\mathrm{BP}$ variability. A decreased slope, moreover, was seen to be associated with a fable baroreceptor sensibility, hence with a declining peripheral cardiovagal function. A similar interpretation has been offered by the "Autonomic Space": a vector parallel to the ordinate axis is called an uncoupled parasympathetic vector, meaning an overwhelming predominance of the vagal tone, while a vector parallel to the abscissa an uncoupled sympathetic one (Berntson et al., 1991, 1993, 1994a, 1994b). Our data may be interpreted accordingly: a steep AARI may be considered to indicate a prevalence of cardiovagal function, while a flat one may indicate a declining cardiovagal with a prevailing sympathetic drive. Since the AARI was only poorly correlated with blood pressure variability, while both AARIs and AARId were highly statistically correlated with heart period variability, it may be concluded that the AARI may be considered an index of cardiovagal function in the 24-h period. This function should be interpreted as the result of the integration between cardiovascular reflexes and central autonomic commands (Silvani, 2008) as confirmed by the circadian day-night blood pressure and HR changes which are determinants of variability (Parati et al., 2003; Silvani, 2008; Schillaci and Parati, 2010). In this connection it may be remarked that the sign of the AARI in the 24 -h period is not consistent with the baroreflex control but rather indicates a prevalence of central autonomic commands.

\subsection{Age effect}

Normal aging is associated with a constant decline of cardiac vagal modulation of heart rate variability (Umetani et al., 1998; Bonnemeier et al., 2003; De Meersman and Stein, 2007; Goff et al., 2010), and by a complex series of changes in the autonomic control of the cardiovascular system, favoring heightened cardiac sympathetic tone with parasympathetic withdrawal and blunted cardiovagal baroreflex sensitivity (Huang et al., 2007; Monahan, 2007; Kaye and Esler, 2008). All these changes appear to level off beyond age 60 years (Low et al., 1997) and reduced heart rate variability is associated with cardiovascular morbidity and mortality (Kaye and Esler, 2008). Our data seems to confirm these previous results. When distributed by decades the AARIs of both NT and HT groups showed a maximum at 20-29 decade followed by a constant decline until the minimum reached at 60-69, reflecting an age-related decline in the cardiovagal function. Hence the AARIs may be viewed as a synthetic index of the central neural regulation of arterial pressure and heart rate in ambulant subjects. More specifically it may be considered a timeindependent index of the circadian day and night 24-h centrally driven sympatho-vagal reciprocity. Although differences in AARIs between hypertensive patients and controls, irrespective of age, were small compared with their average values, what AARI suggests is that life style changes and pharmacological treatments should be directed not only to decrease BP but also to maintain and, when possible, to increase R-R variability. Together with the average values of day and night BP and HR and the absolute value of AASI (Li et al., 2006; Li et al., 2006) the AARIs may thus become a useful index to guide life style changes and pharmacological treatment towards optimum results (Logan, 2010). Recently Pizzinelli et al., in a retrospective study of 676 ABPM quantified the $\mathrm{R}-\mathrm{R}$ interval-systolic blood pressure relation using a non-parametric method to yield a measure of the slope, called the "theta index". Although not quantitatively comparable with AARI, qualitatively similar results have been reported, which is to say that the "theta index" was steeper in the young and in the normotensive group (Pizzinelli et al., 2009). 


\subsection{Hypertension effect}

In the phase-space domain the AARIs is a good index of individual variability. It has been used for the first time, by Coats et al., to study the correlation between heart period and systolic blood pressure, measured invasively, in 142 untreated hypertensive patients (Coats et al., 1991). Being our results comparable with those of Coats et al., the non-invasive approach of ABPM seems to offer a valid alternative method to study the complex autonomic regulation of cardiovascular function in a 24-h period. Overall the comparison between NT and HT groups yielded a highly statistically significant difference for both AARIs and AARId (Fig. 3). When distributed by decades the mean steepness of the regression slope of the NT decades was higher than that of HT decades, this difference not reaching statistical significance, however. This disappointing unexpected result seems to be mainly dependent on the small number of subjects participating to the younger decades and on the difference in gender composition of NT and HT groups which might have contributed to underscore the hypertension effects. Restricting our final consideration to the overall comparison, however, the phase-space distribution of ABPM data seems to confirm the presence of a stronger sympathetic and of a less stable vagal drive in the hypertensive than in the normotensive group.

\subsection{Study limitations}

The relative small number of participants poses limitations on this study, namely that the two groups are composed of male and female patients in different proportions and gender effects could not be quantified. While female subjects were the majority in the normotensive cohort, males were more numerous in the hypertensive group. As shown in the previous study, the phase-space distribution of females was less negative (less steep) than that of males, however (Recordati and Zanchetti, 2008). Thus an unbalanced gender distribution might have contributed to underscore differences between the two groups but not to invalidate the final results.

In the present study the activity level during the 24 -h period has not been standardized. It cannot be excluded, therefore, that decreases in activity level with aging may have contributed to the observed age-related decline in AARI. Differences in activity level would not explain, however, the recorded differences in AARI between the NT and HT decades. In conclusion in the present paper the AARI is proposed as a synthetic index of the prevailing central autonomic balance in the 24 -h period. Although the relation between heart period and blood pressure depends on complex and numerous factors, the representation of ABPM data in the phase-space domain seems sensible enough to unmask the neurally-mediated components and how they are affected by aging and hypertension. In addition of being a useful pathophysiological tool the AARI, being based on both $\mathrm{BP}$ and R-R interval values, may become a reference to guide life style changes and pharmacological therapy towards optimization.

\section{Acknowledgment}

The expert statistical support by Dr C. Galeone (Institute of Medical Statistics and Biometry, University of Milan) is gratefully acknowledged.

\section{References}

Altman, D.G., Machin, D., Bryant, T.N., Gardner, M.J., 2000. Statistics with confidence. Confidence intervals and statistical guidelines, BMJ Book, BMA House, London, UK. Italian Translation, 2004, II Ed. Edizioni Minerva Medica, Torino, Italy.
Berntson, G.G., Cacioppo, J.T., Quigley, K.S., 1991. Autonomic determinisms: the modes of autonomic control, the doctrine of autonomic space, and the laws of autonomic constraints. Psychol. Rev. 98, 459-487.

Berntson, G.G., Cacioppo, J.T., Quigley, K.S., 1993. Cardiac psychophysiology and autonomic space in humans: empirical perspectives and conceptual implications. Psychol. Bull. 114, 296-322.

Berntson, G.G., Cacioppo, J.T., Quigley, K.S., Fabro, V.T., 1994a. Autonomic space and psychophysiological response. Psychophysiol. 31, 44-61.

Berntson, G.G., Uchino, B.N., Cacioppo, J.T., 1994b. Origins of baseline variance and the law of initial values. Psychophysiol. 31, 204-210.

Bertinieri, G., Di Rienzo, M., Cavallazzi, A., Ferrari, A.U., Pedotti, A., Mancia, G., 1988. Evaluation of baroreceptor reflex by blood pressure monitoring in unanesthetized cats. Am. J. Physiol. 254, H377-H383.

Bonnemeier, H., Wiegand, U.K.H., Brandes, A., Kluge, N., Katus, H.A., Richardt, G., Potratz, J., 2003. Circadian profile of cardiac autonomic nervous modulation in healthy subjects: differing effects of aging and gender on heart rate variability. J. Cardiovasc. Electrophysiol. 14, 791-799.

Coats, A.J.S., Conway, J., Sleight, P., Meyer, T.E., Somers, V.K., Floras, J.S., Vann, Jones J., 1991. Interdependence of blood pressure and heart period regulation in mild hypertension. Am. J. Hypertens. 4, 234-238.

Curran-Everett, D., 2000. Multiple comparisons: philosophies and illustrations. Am. J. Physiol. Regul. Integr. Comp. Physiol. 279, R1-R8.

De Meersman, R.E., Stein, P.K., 2007. Vagal modulation and aging. Biol. Psychol. 74, 165-173.

Dolan, E., Thijs, L., Li, Y., Atkins, N., McCormack, P., McClory, S., O'Brien, E., Staessen, J.A., Stanton, A.V., 2006. Ambulatory arterial stiffness index as a predictor of cardiovascular mortality in the Dublin outcome study. Hypert. 47, 365-370.

Furlan, R., Guzzetti, S., Crivellaro, W., Dassi, S., Tinelli, M., Baselli, G., Cerutti, S., Lombardi, F., Pagani, M., Malliani, A., 1990. Continuous 24-hour assessment of the neural regulation of systemic arterial pressure and RR variabilities in ambulant subjects. Circulation 81, 537-547.

Glantz, S.A., 2005. Primer on Biostatistics, 6th Ed. McGraw Hill, New York.

Goff, E.A., Nicholas, C.L., Malaweera, A.S., Simonds, A.K., Trinder, J., Morrell, M.J., 2010. The influence of age on heart rate variability during morning wakefulness. Clin. Auton. Res. 20, 175-182.

Huang, C.-C., Sandroni, P., Sletten, D.M., Weigand, S.D., Low, P.A., 2007. Effect of age on adrenergic and vagal baroreflex sensitivity in normal subjects. Muscle Nerve 36, 637-642.

Kaye, D.M., Esler, M.D., 2008. Autonomic control of the aging heart. NeuroMol. Med. 10, 179-186.

Li, Y., Wang, J.-G., Dolan, E., Gao, P.-J., Guo, H.-F., Nawrot, T., Stanton, A.V., Zhu, D.-L., O'Brien, E., Staessen, J.A., 2006. Ambulatory Arterial Stiffness Index derived from 24-hour ambulatory blood pressure monitoring. Hypert. 47, 359-364.

Logan, A.G., 2010. Ambulatory blood pressure monitoring: its time to move on! J. Hypertens. 28, 2000-2002.

Low, P.A., Denq, J.-C., Opfer-Gehrking, T., Dyck, P.J., O'Brien, P.C., Slezak, J.M., 1997. Effect of age and gender on sudomotor and cardiovagal function and blood pressure response to tilt in normal subjects. Muscle Nerve 20,1561-1568.

Mancia, G., Parati, G., Pomidossi, G., Casadei, R., Di Rienzo, M., Zanchetti, A., 1986. Arterial baroreflexes and blood pressure variabilities in humans. Hypert 8, $147-153$.

Mancia, G., De Backer, G., Dominiczak, A., Cifkova, R., Fagard, R., Germano, G., Grassi, G., Heagerty, A.M., Kjeldsen, S.E., Laurent, S., Narkiewicz, K., Ruilope, L., Rynkiewicz, A., Schmieder, R.E., Struijker Boudier, H.A., Zanchetti, A., 2007. Guidelines for the management of arterial hypertension of the European Society of Hypertension (ESH) and of the European Society of Cardiology (ESC). J. Hypertens. 25, 1105-1187.

Monahan, K.D., 2007. Effect of aging on baroreflex function in humans. Am. J. Physiol. Regul. Integr. Comp. Physiol. 293, R3-R12.

Parati, G., Di Rienzo, M., Mancia, G., 2000. How to measure baroreflex sensitivity: from the cardiovascular laboratory to daily life. J. Hypertens. 18, 7-19.

Parati, G., Bilo, G., Vettorello, M., Groppelli, A., Maronati, A., Tortorici, E., Caldara, G., Mancia, G., 2003. Assessment of overall blood pressure variability and its different components. Blood Press. Monit. 8, 155-159.

Pizzinelli, P., Iellamo, F., Beltrami, S., Lucini, D., Pagani, M., 2009. Time-independent indices of circadian blood pressure and heart rate regulation from ambulatory blood pressure monitoring. J. Hypertens. 27, 1178-1185.

Recordati, G., 2003. A thermodynamic model of the sympathetic and parasympathetic nervous systems. Autonom. Neurosci.: Basic \& Clinical 103, 1-12.

Recordati, G., Zanchetti, A., 2008. The 24 h blood pressure-R-R interval relation in ambulatory monitoring. Autonom. Neurosci.: Basic \& Clinical 139, 68-77.

Schillaci, G., Parati, G., 2010. Deteminants of blood pressure variability in youth: at the roots of hypertension. J. Hypertens. 28, 660-664.

Silvani, A., 2008. Physiological sleep-dependent changes in arterial blood pressure: central autonomic commands and baroreflex control. Clin. Exp. Pharmacol. Physiol. 35, 987-994.

Umetani, K., Singer, D.H., McCraty, R., Atkinson, M., 1998. Twenty-four hour time domain heart rate variability and heart rate: relations to age and gender over nine decades. J. Am. Coll. Cardiol. 31, 593-601.

Wallin, G., Charkoudian, N., 2007. Sympathetic neural control of integrated cardiovascular function: insights from measurement of human sympathetic nerve activity. Muscle Nerve 36, 595-614. 